\title{
Sintomas somáticos inexplicados clinicamente: um campo impreciso entre a psiquiatria e a clínica médica
}

\author{
Unexplained somatic symptoms: an undefined field betwen psychiatry and \\ internal medicine
}

José Atílio Bombana

\begin{abstract}
Resumo
Quadros clínicos caracterizados por sintomas somáticos inexplicados devido a condições médicas gerais são muito freqüentes na prática médica e representam, em geral, um quarto a metade dos atendimentos em ambos os cuidados - primários e secundários. Atualmente são classificados na psiquiatria como transtornos somatoformes (TSs) e na clínica médica, como síndromes somáticas funcionais (SSFs). A categoria diagnóstica dos TSs tem sido questionada, suscitando proposta para sua extinção nas futuras classificações internacionais. As SSFs caracterizam-se mais por sintomas, sofrimento e incapacidade do que por patologias específicas, e incluem fibromialgia, síndrome do intestino irritável (SII), síndrome da fadiga crônica, várias síndromes dolorosas, entre outras. A sobreposição dos quadros clínicos leva ao questionamento da existência de um ou vários diagnósticos, tanto entre diferentes SSFs como entre elas e os TSs, apontando também para a questão da co-morbidade. Um mesmo paciente, ao ser atendido por um psiquiatra, pode receber um diagnóstico de TS, mas, se encaminhado para um clínico, poderia receber o diagnóstico de SSF. Apresenta-se um campo impreciso, sugerindo, portanto, que deverão ocorrer modificações em termos de conceitualização, classificação diagnóstica e abordagem terapêutica. 0 estudo dos sintomas somáticos inexplicados clinicamente demonstra a necessidade de abordagens integradas. São mencionadas algumas experiências nesse campo do Programa de Atendimento e Estudos de Somatização da Universidade Federal de São Paulo (UNIFESP).

Palavras-chave: transtornos somatoformes, fibromialgia, diagnóstico, co-morbidade.
\end{abstract}

\begin{abstract}
Clinical features characterized by symptoms unexplained by general medical conditions are very common in the clinical practice, accounting for a quarter to half of visits in both primary and secondary medical care facilities. They are currently classified as somatoform disorders (SD) in psychiatry, and as functional somatic syndromes (FSS) in Internal medicine. The diagnostic category of SD has been questioned, and its exclusion has even been proposed in future international classifications. FSS are characterized by symptoms, suffering and incapability rather than by specific diseases, and include but are not limited to fibromyalgia, irritable bowel syndrome, chronic fatigue syndrome, and several pain syndromes. Overlapping of clinical features raises the question of whether one or several diagnoses are present, both among different FSS and between FSS and SD, also pointing to the comorbidity issue. The same patient may be diagnosed with SD when seen by a psychiatrist, but when referred to a general practitioner he/she could be diagnosed with FSS. An overlapping field is presented, suggesting that modifications should occur in terms of conceptualization, diagnostic classification and therapeutic approach. The study of medically unexplained symptoms demonstrates the need for integrated approaches. Some related experiences of the Program of Care and Studies on Somatization (Universidade Federal de São Paulo [UNIFESP]) are presented.

Key words: somatoform disorders, fibromyalgia, diagnosis, comorbidity.
\end{abstract}

Universidade Federal de São Paulo (UNIFESP) (Bombana JA)

Recebido
16-11-06
Aprovado
18-12-06

Correspondência para: José Atílio Bombana

Departamento de Psiquiatria da Unifesp - Rua Botucatu, 740/3 andar - Ambulatório - 04023-900 - São Paulo-SP - Tel./Fax: (011) 3887-1797 - e-mail: bombana@uol.com.br 


\section{Introdução}

Quadros clínicos caracterizados por sintomas somáticos não explicados por condições médicas gerais têm sido continuamente relatados ao longo da história da medicina. Esses quadros também apresentam comumente incapacitação desproporcional aos achados do exame físico, inexistência de anormalidades laboratoriais e aparente associação com fatores psicossociais e/ou estresse, que representam um quarto a metade dos atendimentos em ambos os cuidados - primários e secundários (Kroenke, 2003; Nimnuan et al., 2001). Mesmo que sua gravidade muitas vezes possa ser questionada, o incômodo e as limitações que produzem justificam sua aceitação como transtornos, embora de difíceis conceitualização e classificação. Tais quadros são conhecidos como somatização, transtornos somatoformes, sintomas somáticos não explicados clinicamente e síndromes funcionais (ou psicossomáticas) (Wessely et al., 1999). Eles representam uma região obscura no conhecimento médico entre a psiquiatria e a clínica médica. Sua classificação reflete essa dificuldade: na psiquiatria são atualmente nomeados como transtornos somatoformes (TSs) e na clínica médica, como síndromes somáticas funcionais (SSFs) (Mayou et al., 2005). Este artigo pretende delinear o estado do conhecimento atual nesse tema relevante.

\section{Um breve histórico}

As primeiras concepções desses quadros recaíram, há cerca de dois milênios, sobre a hipótese de distúrbios de órgãos, em particular do útero, sendo os mesmos chamados de histéricos. O manejo clínico ocorria por meio de ungüentos vaginais e manipulação direta do órgão. Somente após a Renascença (século XV) passaram a ser explicados como doenças do sistema nervoso. No final do século XVII, fatores psicológicos começaram a ser considerados na sua etiologia. Entretanto, no início do século XVIII, além de estímulos a exercícios físicos e das mudanças no contexto social, a investigação do estado mental dos pacientes e a promoção de esperança e otimismo eram propostas. No século XIX houve um retorno das explicações predominantemente orgânicas. Como as investigações anatomopatológicas não evidenciavam anormalidades, os quadros foram chamados de funcionais, sendo propostas intervenções físicas. Ao final desse século, por certo período esses quadros foram concebidos como distúrbios funcionais reversíveis do sistema nervoso, e seu tratamento incluía atenção a aspectos psicológicos durante 0 atendimento médico. Já no início do século XX surgiu a concepção da origem exclusivamente psicológica ou mental, difundindo-se então a idéia de psicogênese e propondo-se um tratamento explicitamente psicológico. A somatização surgiu como explicação das manifestações de sintomas somáticos devido a conflitos psíquicos (Sharpe e Carson, 2001).

As abordagens mais recentes tratam de duas situações: numa estão os pacientes acompanhados por clínicos, que, quando encaminhados aos psiquiatras, resistem à indicação, recebendo um tratamento médico tradicional e freqüentemente com muitas solicitações de exames e múltiplos encaminhamentos para especialistas. Na outra estão os pacientes acompanha- dos em serviços de psiquiatria, que recebem tratamento medicamentoso (principalmente com antidepressivos e ansiolíticos) elou psicoterapia (O'Malley et al., 1999; Allen et al., 2002).

Nota-se uma oscilação entre os pólos orgânico e psíquico como suposta origem desses quadros ao longo do tempo.

\section{A psiquiatria e os transtornos somatoformes}

Nos serviços de psiquiatria esses pacientes são atualmente diagnosticados como portadores de TSs, cujo conceito é de um grupo de transtornos que se caracterizam pela presença, por longo tempo (meses ou anos), de queixas freqüentes de sintomatologia física sugestivas da existência de substrato orgânico, mas que não são totalmente explicadas por nenhuma doença orgânica conhecida, sendo atribuídas, em seu desencadeamento e manutenção, a fatores e conflitos psicológicos (WHO, 1993; American Psychiatric Association, 2002).

Os somatizadores são responsáveis por um número desproporcionalmente alto de consultas médicas, gerando excessivos gastos no sistema de saúde. Dados americanos apontam que até $50 \%$ dos custos de ambulatórios médicos são a eles atribuídos (Lipowski, 1988; Barsky e Klerman, 1983).

$\mathrm{Na} 10^{\mathrm{a}}$ revisão da Classificação Estatística Internacional de Doenças e Problemas Relacionados à Saúde (CID-10) (WHO, 1993) e no texto revisado da quarta revisão do Manual Diagnóstico e Estatístico Transtornos Mentais (DSM-IV-TR) (American Psychiatric Association, 2002) são descritos sete diferentes tipos de transtorno somatoforme:

- transtorno de somatização: caracterizado por uma longa história (mais de dois anos) de múltiplos sintomas físicos, afetando vários sistemas orgânicos, mutáveis ao longo do tempo, numa evolução crônica flutuante e marcada por inúmeras, tentativas de tratamento médico, geralmente ineficazes. O funcionamento social tende a ser precário e as atividades profissionais, limitadas. Há um grande predomínio de mulheres, e o início do quadro tende a ser precoce;

- transtorno somatoforme indiferenciado: quadro semelhante ao anterior, mas que não preenche todas as características descritas. As queixas podem ocorrer em número restrito, ou o tempo de evolução ser curto, ou, ainda, não ocorrerem comprometimentos sociais e profissionais. Esse quadro ganha importância à medida que apresentações completas (como no transtorno de somatização) são menos freqüentes, enquanto essas menos severas são muito mais comuns (Kroenke et al., 1997);

- transtorno hipocondríaco: seu aspecto básico é uma interpretação errônea de sensações corporais corriqueiras, que são percebidas como anormais, levando ao medo e à crença de se estar gravemente doente. O curso é normalmente crônico e flutuante. Há uma forte resistência em aceitar o diagnóstico e a conduta propostos pelo médico;

- disfunção autonômica somatoforme: caracteriza-se por queixas objetivas decorrentes da excitação do sistema nervoso autônomo (como palpitações, sudorese e tremores), que cursam juntamente com sintomas subjetivos e inespecíficos (como sensações de dor, aperto ou ardor 
incaracterísticos) e que despertam preocupação quanto a um possível comprometimento de um órgão e/ou sistema específicos. Freqüentemente esses quadros são genérica e pejorativamente chamados de distonia neurovegetativa (DNV) (Bombana et al., 1997);

- transtorno doloroso somatoforme persistente: quadro de dor persistente e intensa não atribuível a patologias médicas conhecidas. Há freqüente associação com sintomas depressivos;

- transtorno conversivo: sintomas ou déficits que afetam os sistemas neuromuscular voluntário e/ou sensórioperceptivo clinicamente inexplicáveis. Consta no DSM-IV$\mathrm{TR}$, enquanto na CID-10 faz parte da categoria transtornos dissociativos (ou conversivos);

- transtorno dismórfico corporal: preocupação com um defeito imaginado ou exagerado na aparência física. Consta no DSM-IV-TR, enquanto na CID-10 está incluído em transtorno hipocondríaco.

\section{Críticas à categoria transtornos somatoformes}

Desde a introdução da categoria diagnóstica TS, inúmeros questionamentos têm sido feitos a seu respeito (Mayou et al., 2005; Kroenke et al., 1997; Fink et al., 2005; Creed e Barsky, 2004), propondo-se até sua extinção na próxima edição do DSM. Alguns pontos controversos são:

- a terminologia é problemática para os pacientes, parecendo que o conceito de somatização questiona a realidade e a genuinidade do sofrimento;

- a categoria é inerentemente dualística: a idéia de que os sintomas somáticos se dividem entre os que representam doença física e os que são psicogênicos é teoricamente questionável;

- TSs não são aceitos em algumas culturas, como na China, onde se utiliza o DSM, mas se exclui essa categoria;

- há sobreposição com outros diagnósticos psiquiátricos, como depressão e ansiedade;

- não há confiabilidade nas subcategorias: o transtorno de somatização, diagnóstico decisivo na criação da categoria, é muito pouco diagnosticado, enquanto o TS indiferenciado, que é pobremente definido, de uma categoria residual passou à categoria mais largamente aplicável;

- existe ambigüidade nos critérios de exclusão: não há clareza quanto a quais diagnósticos médicos deveriam ser vistos como excludentes, como, por exemplo, as SSFs;

- TSs causam confusão quanto a questões médicolegais, de seguros e licenças.

\section{A clínica médica e as síndromes somáticas funcionais}

Nos serviços de clínica médica, quadros semelhantes freqüentemente são classificados como SSFs (ou psicossomáticas). Esses termos têm sido utilizados para designar inúmeras síndromes relacionadas, caracterizadas mais por sintomas, sofrimento e incapacidade do que por doenças específicas ou anormalidades demonstráveis de estrutura ou função (Barsky e Borus, 1999). Essas síndromes incluem, entre outras, fibromialgia, síndrome do intestino irritável (SII), síndrome da fadiga crônica, sensibilidade química múltipla, lesão por esforço repetitivo/distúrbios osteomusculares relacionados ao trabalho (LER/DORT), hipoglicemia, precordialgia não-cardíaca, transtorno temporomandibular e várias síndromes dolorosas (Wessely et al., 1999; Barsky e Borus, 1999; Aaron e Buchwald, 2001).

Parece haver características clínicas, comportamentais, epidemiológicas e fisiológicas semelhantes nesses quadros. Muitos pacientes diagnosticados com uma das síndromes também preenchem diagnóstico para outra(s) dessas síndromes (Wessely et al., 1999; Aaron et al., 2000). Embora complexas, pobremente compreendidas e heterogêneas, as SSFs têm o suficiente em comum para justificar uma discussão sobre elas, talvez como variantes de um processo biopsicossocial comum. Alguns autores propõem uma fisiopatologia comum (Barsky e Borus, 1999).

O diagnóstico de um paciente pode ser fortemente influenciado pelo contexto e pela especialidade médica de quem o faz, assim como pela proeminência de alguns sintomas do paciente. Portanto os polissintomáticos podem receber inúmeros diagnósticos distintos. Pacientes com SSFs têm taxas elevadas de transtornos psiquiátricos, particularmente ansiedade, depressão e TS. A grande prevalência de quadros somatoformes sugere uma tendência preexistente a experienciar e referir desconforto corporal. Também aqui existe dificuldade para uma inserção nosológica. Essas síndromes são freqüentemente refratárias aos tratamentos médicos comuns e às medidas paliativas habituais. A ineficiência do tratamento nesses pacientes, ao invés de causar seu abandono como seria o mais comum, leva a uma intensificação do mesmo (Barsky e Borus, 1999).

\section{Um ou vários diagnósticos?}

A sobreposição de diagnósticos leva ao questionamento da existência de vários fenômenos clínicos distintos ou de discretas diferenças de apresentação de uma única entidade (Wessely et al., 1999; Sharpe e Carson, 2001). E isso se coloca tanto para a sobreposição entre diferentes SSFs, como entre elas e os TSs, o que suscita também a questão da co-morbidade. $O$ agrupamento de síndromes funcionais parece ser, em parte, em razão da severidade do transtorno emocional e, em outra parte, por uma tendência do indivíduo a reagir a sobrecargas com alterações fisiológicas em vários sistemas ou com somatização, ou ambos.

Alguns autores consideram ser mais vantajoso conceitualizar um paciente dito somatizador como alguém em quem se agruparam inúmeras síndromes funcionais (Kellner, 1994). Alguns estudos têm procurado especificar o grau de interação entre esses quadros, buscando identificar um possivel fator geral responsável em grande parte pelas SSFs e pelos TSs (Robbins et al., 1997; Deary, 1999). A Figura apresenta uma possível representação gráfica para a sobreposição dos diagnósticos das SSFs e dos TSs. 
Figura. Sobreposição de diagnósticos das SSF e dos TSs e possível relação com um fator geral

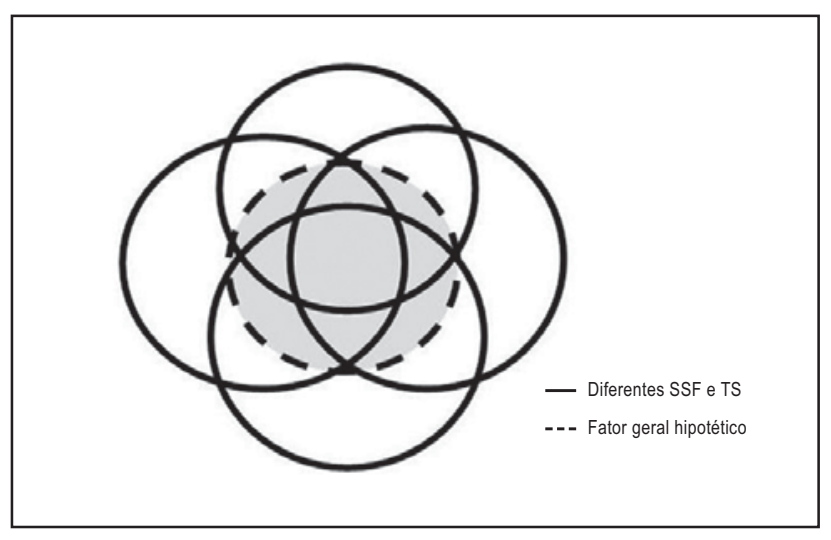

O resultado de tudo isso é que um mesmo paciente, se atendido por um psiquiatra, pode ser visto como somatizador e receber o diagnóstico de TS, mas, se for encaminhado para um clínico, pode receber o diagnóstico de SSF (Kellner, 1994; Sharpe, 2002).

A observação por mais de dez anos dos pacientes do Programa de Atendimento e Estudos de Somatização do Departamento de Psiquiatria da Universidade Federal de São Paulo (Unifesp), destinado ao atendimento de somatizadores, revela uma ocorrência freqüente de quadros somáticos e de atendimentos múltiplos em diferentes especialidades (Bombana et al., 2000). Eles freqüentam os ambulatórios do Hospital São Paulo, assim como de outros locais, com uma intensidade variável de caso para caso, mas que, no geral, transmitem forte impressão de superutilização dos serviços médicos. Apresentam quadros clínicos sem alterações demonstráveis de estrutura ou função isoladamente ou, por vezes, mesclados com patologias específicas. Esses pacientes foram avaliados numa pesquisa em que se investigou a presença de critérios diagnósticos para fibromialgia.

Foram pesquisados 23 pacientes (19 mulheres e quatro homens) e, desse total, $73,9 \%$ deles preencheram os critérios diagnósticos. Nesse mesmo estudo, o Ambulatório de Transtornos Afetivos da Unifesp também foi pesquisado, tendo sido examinados 91 pacientes (68 mulheres e 23 homens), com $14,3 \%$ do total preenchendo os critérios diagnósticos (comunicação pessoal de pesquisa inédita pela Dra. Cinthia Yoshimura Hsu e pelo professordoutor Daniel Feldman, do Departamento de Reumatologia da UNIFESP). A expressiva porcentagem de fibromialgia entre os somatizadores e a comparação com os dados do outro serviço sugerem a importância da sobreposição de diagnósticos entre os primeiros.

\section{Conclusão}

O conteúdo apresentado revela um campo impreciso, sugerindo que modificações deverão ocorrer em termos de conceitualização, classificação diagnóstica e abordagem terapêutica. Quanto à classificação, uma possibilidade é o aperfeiçoamento dos sistemas atuais, com correções dos principais itens questionados, mas que conservaria a categoria TS (Henningsen e Löwe, 2006). Numa outra direção propõe-se a abolição dessa categoria, promovendo-se alterações mais radicais nas classificações e uma redistribuição dos diagnósticos (Mayou et al., 2005).

Nesse caso sugere-se que:

- os quadros somáticos fortemente associados à depressão e/ou à ansiedade passem a figurar nessas categorias (como depressão e/ou ansiedade somáticas). A hipocondria também se enquadraria num caso particular de ansiedade (voltada para o próprio corpo);

- o transtorno de somatização seria classificado como uma combinação de transtorno de personalidade mais um transtorno de ansiedade ou afetivo;

- as síndromes funcionais e o transtorno doloroso seriam alocados num eixo classificatório que abordasse as condições médicas gerais (como o eixo III do DSM).

Quanto à abordagem terapêutica, alguns autores propõem uma consulta médica psicologicamente enriquecida, com a incorporação de técnicas psicoterápicas básicas, ou seja, uma tentativa de integrar princípios dos tratamentos psiquiátrico e psicológico aos cuidados médicos (Wessely et al., 1999; Sharpe e Carson, 2001). Ainda nesse quesito outra vertente a ser considerada é a psicossomática (ou medicina psicossomática), a qual propõe uma visão multidisciplinar e de integração, enfocando o paciente como ser histórico, um sistema único constituído por corpo, mente e meio social (Mello Filho, 1992).

O campo dos sintomas somáticos não explicados clinicamente demonstra a necessidade de abordagens integradas. Em nosso programa (na UNIFESP) temos optado por uma que contempla tanto a aproximação com os clínicos, quanto uma proposta de atendimento psicoterápico acrescido de tratamento medicamentoso, quando indicado (Bombana et al., 2000).

O reconhecimento dos impasses aponta para a necessidade de novos estudos, principalmente os longitudinais com amostras da população geral, ou de cuidados primários (Creed e Barsky, 2004).

\section{Agradecimentos}

À Dra. Cinthia Yoshimura Hsu e ao professor-doutor Daniel Feldman, do Departamento de Reumatologia da UNIFESP, pelo gentil fornecimento dos dados da pesquisa. 


\section{Referências}

Aaron LA, Burke MM, Buchwald D. Overlapping conditions among patients with chronic fatigue syndrome, fibromyalgia, and temporomandibular disorder. Arch Intern Med, 160: 221-7, 2000

Aaron $\mathrm{LA}$, Buchwald $\mathrm{D}$. A review of the evidence for overlap among unexplained clinical conditions. Ann Intern Med, 134(9): 868-81, 2001.

Allen LA, Escobar JI, Lehr PM, Gara MA, Woolfolk RL. Psychosocial treatments for multiple unexplained physical symptoms: a review of the literature. Psychosom Med, 64: 939-50, 2002.

American Psychiatric Association. Manual diagnóstico e estatístico de transtornos mentais. 4 ed. Texto revisado DSM-IV-TR. Porto Alegre: Artmed; 2002

Barsky AJ, Klerman GL. Overview: hypochondriasis, bodily complaints and somatic styles. Am J Psychiatry, 140(3): 273-83, 1983.

Barsky AJ, Borus JF. Functional somatic syndromes. Ann Intern Med, 130: 910-21, 1999

Bombana JA, Ferraz MPT, Mari JJ. Neurovegetative dystonia: psychiatric evaluation of 40 patients diagnosed by general physicians in Brazil. J Psychosom Res, 43(5): 489-95, 1997.

Bombana JA, Leite ALSS, Miranda CT. Como atender aos que somatizam? Descrição de um programa e relatos concisos de casos. Rev Bras Psiquiatr, 22(4): 180-4, 2000.

Creed F, Barsky A. A systematic review of the epidemiology of somatization disorder and hypochondriasis. J Psychosom Res, 56: 391-408, 2004.

Deary IJ. A taxonomy of medically unexplained symptoms. J Psychosom Res, 47(1): 51-9, 1999.

Fink P, Rosendal M, Olesen F. Classification of somatization and functional somatic symptoms in primary care. Aust NZ J Psychiatry, 39(9): 77281, 2005.

Henningsen P, Löwe B. Depression, pain, and somatoform disorders. Curr Opin Psychiatry, 19(1): 19-24, 2006.

Kellner R. Psychosomatic syndromes, somatization and somatoform disorders. Psychother Psychosom, 61: 4-24, 1994.
Kroenke K, Spitzer RL, deGruy III FV, Hahn SR, Linzer M, Williams JBW, Brody D, Davies M. Multisomatoform disorder - an alternative to undifferentiated somatoform disorder for the somatizing patient in primary care. Arch Gen Psychiatry, 54: 352-8, 1997.

Kroenke K. Patients presenting with somatic complaints: epidemiology, psychiatric comorbidity and management. Int J Methods Psychiatr Res, 12: 34-43, 2003.

Lipowski ZJ. Somatization: the concept and its clinical application. Am J Psychiatry, 145: 1358-68, 1988.

Mayou R, Kirmayer LJ, Simon G, Kroenke K, Sharpe M. Somatoform disorders: time for a new approach in DSM-V. Am J Psychiatry, 162(5): 847-55, 2005.

Mello Filho J. Introdução. In: Mello Filho J, editor. Psicossomática hoje. Porto Alegre: Artes Médicas, 1992, p. 19-27.

Nimnuan C, Hotopf M, Wessely S. Medically unexplained symptoms. An epidemiological study in seven specialities. J Psychosom Res, 51: 361-7, 2001.

O'Malley PG, Jackson JL, Santoro J, Tomkins G, Balden E, Kroenke K. Antidepressant therapy for unexplained symptoms and symptom syndromes. J Fam Pract, 48: 980-90, 1999.

Robbins JM, Kirmayer LJ, Hemami S. Latent variable models of functional somatic distress. J Nerv Ment Dis, 185(10): 606-15, 1997

Sharpe M, Carson A. "Unexplained" somatic symptoms, functional syndromes, and somatization: do we need a paradigm shift? Ann Intern Med, 134(9): 926-30, 2001.

Sharpe M. Medically unexplained symptoms and syndromes. Clin Med, 2(6): 501-4, 2002

Wessely S, Nimnuan C, Sharpe M. Functional somatic syndromes: one or many? Lancet, 354: 936-9, 1999.

World Health Organization. Classificação de transtornos mentais e de comportamento da CID-10. Porto Alegre: Artes Médicas; 1993. 